\title{
Electronics of an ion trap with integrated time-of-flight mass spectrometer
}

\author{
Christian Schneider, ${ }^{1, *}$ Steven J. Schowalter, ${ }^{1}$ Peter $\mathrm{Yu},{ }^{1}$ and Eric R. Hudson ${ }^{1}$ \\ ${ }^{1}$ Department of Physics and Astronomy, University of California, Los Angeles, California 90095, USA
}

\begin{abstract}
Recently, we reported an ion trap experiment with an integrated time-of-flight mass spectrometer (TOFMS) [1] focussing on the improvement of mass resolution and detection limit due to sample preparation at millikelvin temperatures. The system utilizes a radio-frequency (RF) ion trap with asymmetric drive for storing and manipulating laser-cooled ions and features radial extraction into a compact $275 \mathrm{~mm}$ long TOF drift tube. The mass resolution exceeds $m / \Delta m=500$, which provides isotopic resolution over the whole mass range of interest in current experiments and constitutes an improvement of almost an order of magnitude over other implementations. In this manuscript, we discuss the experimental implementation in detail, which is comprised of newly developed drive electronics for generating the required voltages to operate RF trap and TOFMS, as well as control electronics for regulating RF outputs and synchronizing the TOFMS extraction.
\end{abstract}

\section{INTRODUCTION}

Experiments with molecular ions in radio-frequency $(\mathrm{RF})$ ion traps have rapidly evolved in physics and chemistry in recent years. Such experiments focus on the production of molecules [2]; their cooling [3-8]; reactions of trapped ions with (untrapped) neutral reactants [912]; spectroscopy of molecular ions [13-17]; and precision measurements [18]. As opposed to neutral molecules, molecular ions allow for easy trapping and, optionally, sympathetic cooling of their translational degrees of freedom with co-trapped, laser-cooled atomic ions [19-21]. Since ion trapping is largely species independent, it is important to have a robust means to identify the trapped ions. This identification can be used to deduce reaction properties, such as reaction rates or branching ratios, or for destructive spectroscopy techniques involving e.g. photo-dissociation.

Closely related are experimental efforts with hybrid atom-ion traps [22-26]. Their implementation typically involves an RF ion trap for confining ions, which are overlapped with a cold cloud of atoms [22, 23] or a BoseEinstein condensate [27, 28]. All-optical hybrid traps for atoms and ions are also in development, in which the $\mathrm{RF}$ trap can be turned off during certain experimental sequences [29-32]. A chief application of these systems is the study of cold/ultra-cold collisions and reactions of ions and atoms [33-40]. Conclusions on the reaction mechanisms can be drawn by trapping and, again, subsequently analysing charged reaction products.

Mass spectrometry (MS) constitutes a powerful and straight-forward way for the analyses of product ion samples in such experiments. While mass spectrometers with resolutions exceeding $m / \Delta m=100,000$ are commercially available [41], such systems are usually bulky, expensive and difficult to integrate in experiments with cold ions and atoms. Hence, various techniques have been used to allow for discriminating different ions species in

* christian.schneider@physics.ucla.edu such experiments. These include mass filtering [42]; resonant excitation of the secular ion motion [20, 43, 44]; laser-induced fluorescence techniques [5, 9, 45-47]; or integrated time-of-flight mass spectrometers (TOFMSs) $[1,12,14,15,18]$. The former techniques are applicable without changes to the vacuum systems, but complicated due to nonlinear resonances of the RF trap [48], complex interpretation of the resulting spectra, demanding requirements on laser cooling including formation of ion Coulomb crystals, and/or the required molecular dynamics simulations. This is in particular true, if the sample contains a variety of potentially unknown ion species. As an alternative, integrated TOFMSs have proven to be unambiguos, be conceptually simple, be widely applicable, and provide a relatively high, sometimes isotopic, mass resolution.

An early implementation of an RF trap with integrated TOFMS is given in Ref. 14 and reaches mass resolutions of typically $m / \Delta m \sim 50$. The linear RF trap is operated symmetrically (with RF voltages of opposite sign at neighboring electrodes) using two center-tapped $\mathrm{RF}$ transformers. Pulsed application of slightly different high voltages $(\mathrm{HVs})$ to the center taps of the transformers creates the two-stage electric field of a Wiley-McLaren TOFMS [49] and extracts ions radially [50, 51] into a TOF drift tube. The radial extraction of this implementation has the advantage of a more compressed sample compared to an extraction along the axis of the RF trap, but sacrifices some of the gain in mass resolution due to the presence of RF ringing during the extraction. This system has been used to perform photo-dissociation spectroscopy of $\mathrm{BaCl}^{+}$[14], $\mathrm{SrCl}^{+}[16]$, and $\mathrm{DyCl}^{+}$[17].

A later TOFMS implementation using a six-rod quadrupole RF trap with radial extraction is described in Ref. 18 and has been used for spectroscopy of $\mathrm{HfF}^{+}$. The $\mathrm{RF}$ trap is driven at an exceptionally low drive frequency of about $50 \mathrm{kHz}$, such that drive voltages are generated without resonant enhancement which facilitates the application of the TOF extraction voltages. Although the six-rod RF trap has a different motivation in this system, such a trap geometry has the potential advantage of being able to use separate electrodes for RF and $\mathrm{HV}$ : 
while two opposing rods can be driven with RF, HVs can be applied to the other four rods and ions are extracted through a gap between two neighboring HV electrodes. A drawback for certain applications would be the reduced optical access for establishing, for example, an atom trap.

Another TOFMS implementation with axial extraction has been used to perform spectroscopy of $\mathrm{AlH}^{+}$[15]. While the mass resolution is limited, the implementation is appealing because of its simplicity and the separation of RF and $\mathrm{HV}$ electrodes.

Recently, a TOFMS with radial extraction has been demonstrated [12], in which the RF trap is operated with digital HV pulses instead of sinusoidal voltages [52, 53]. This concept appears compelling, because the abscence of RF voltages prevents the associated ringing and digital $\mathrm{HV}$ pulses can be directly reused for extraction into the TOFMS, however, the mass resolution in the given implementation remains $m / \Delta m \leq 90$. Further, the digital drive leads to enhanced micromotion and increased requirements on phase matching between different electrodes, which is disadvantageous during experimental cycles requiring cold ion samples.

In 2014, we demonstrated an RF trap with integrated TOFMS [1], which is loosely based on Refs. 54 and 14. The TOFMS has a significantly higher mass resolution $(m / \Delta m>500)$ than other implementations. The $\mathrm{RF}$ trap is operated asymmetrically and can be actively damped during the application of the $\mathrm{HV}$ extraction pulses to prevent ringing. The basic setup and the effect of laser cooling on mass spectrometry has been sketched in Ref. 1; here, we focus on the technical details.

In the following, we first explain the electronics for the RF trap/TOFMS in Sec. II. This section is subdivided into an overview of the experimental apparatus (Sec. II A), the description of drive units generating the RF voltages and HVs (Sec. II C) and a control unit (Sec. IID), and remarks on the wiring of the RF trap and measuring of the voltages (Sec. II E). Subsequently, in Sec. III, we conclude with a brief discussion of the performance of the system (Sec. III A) and potential future improvements (Sec. III B).

\section{EXPERIMENTAL IMPLEMENTATION}

\section{A. Overview}

The apparatus consists of a segmented linear RF trap, and a basic, Wiley-McLaren [49] type TOFMS (see Fig. 1). More details and a three-dimensional rendering of the relevant components are given in Ref. 1. Briefly, the $\mathrm{RF}$ trap is driven asymmetrically with one pair of diagonally opposing electrodes at $\mathrm{RF}$ voltage (amplitude $\left.V_{\mathrm{RF}}=V_{0}\right)$ and the other pair at $\mathrm{RF}$ ground $\left(V_{\mathrm{RF}}=0\right)$. We can choose between a drive frequency $\Omega$ of either $\Omega_{<} \approx 2 \pi \times 720 \mathrm{kHz}$ or $\Omega_{>} \approx 2 \pi \times 1.8 \mathrm{MHz}$ depending on the pair of electrodes being driven and reach $\mathrm{RF}$ amplitudes of up to $V_{0} \approx 750 \mathrm{~V}$.

For the extraction into the TOF drift tube, the RF voltages are quickly turned off. Subsequently, the electrodes are pulsed to DC HVs $U_{\mathrm{HV}}$ with a $10 \%-90 \%$ rise time of $\approx 250 \mathrm{~ns}$. The HV is applied such that a twostage electric field is established [49], which radially extracts the cold atoms and molecules from the RF trap into the TOFMS [50, 51]. This is accomplished by applying a slightly lower $\mathrm{HV}$ to the electrodes which are closer to the TOFMS $\left(U_{\mathrm{HV}}=U_{1}=1.2 \mathrm{kV}\right)$ than to the ones that are farther $\left(U_{\mathrm{HV}}=U_{2}=1.4 \mathrm{kV}\right)$.

This scheme leads to a noticable complication: While the same $\mathrm{RF}$ voltage $V_{\mathrm{RF}}$ is required on diagonally opposing electrodes for trapping, different $U_{\mathrm{HV}}$ must be applied to these electrodes for extraction into the TOFMS. In total, four different configurations of voltages $\left(V_{\mathrm{RF}} ; U_{\mathrm{HV}}\right)$ must be generated: $\left(0 ; U_{1}\right),\left(0 ; U_{2}\right),\left(V_{0} ; U_{1}\right)$, and $\left(V_{0} ; U_{2}\right)$. Additionally, different low-voltage $\mathrm{DC}$ voltages $U_{\mathrm{DC}}$ need to be superimposed with $V_{\mathrm{RF}}$ on some segments to compensate micromotion and provide axial confinement.

The developed circuit, referred to as the drive unit (see Sec. II C) in what follows, generates one triplet of voltages $\left(V_{\mathrm{RF}} ; U_{\mathrm{HV}} ; U_{\mathrm{DC}}\right)$. We typically drive each segment of the RF trap with its own drive unit such that a total of twelve copies of these drive units is required. This choice has the benefit of a large degree of freedom in the applied voltages, but comes at the price of a relatively high complexity for matching both the RF voltages (frequency, phase and amplitude) and HV pulses (timing, slope and amplitude). A control unit (see Sec. IID) outputting up to 16 synchronized RF signals with adjustable frequency, phase and amplitude and incorporating synchronization circuits plays a key role in this matching. In principle, the number of drive units could be reduced to four, if a non-segmented RF trap with external DC electrodes was used.

\section{B. RF Trap and TOFMS Design}

The design of both RF trap and TOFMS (see Fig. 1) is based on Ref. 14, which contains a detailled analysis and optimization of relevant parameters (such as extraction voltages or mechanical dimensions) using both analytical formulas for a Wiley-McLaren type TOFMS [49] and ion optical simulations. In summary, the radial extraction from the RF trap leads to a defocussing of the ions in the $z$ direction due to the electric field inhomogeneities from the RF trap electrodes. To compensate for this, the first Einzel lens at the beginning of the drift tube focusses the ions. As the component of the extraction field in the $y$ direction is close to zero (for symmetry reasons), the ions do not experience a defocussing in the $y$ direction resulting in astigmatism, whose effects are mitigated by the second Einzel lens. The skimmer in front of the first Einzel lens prevents ions with large deflection angles to 


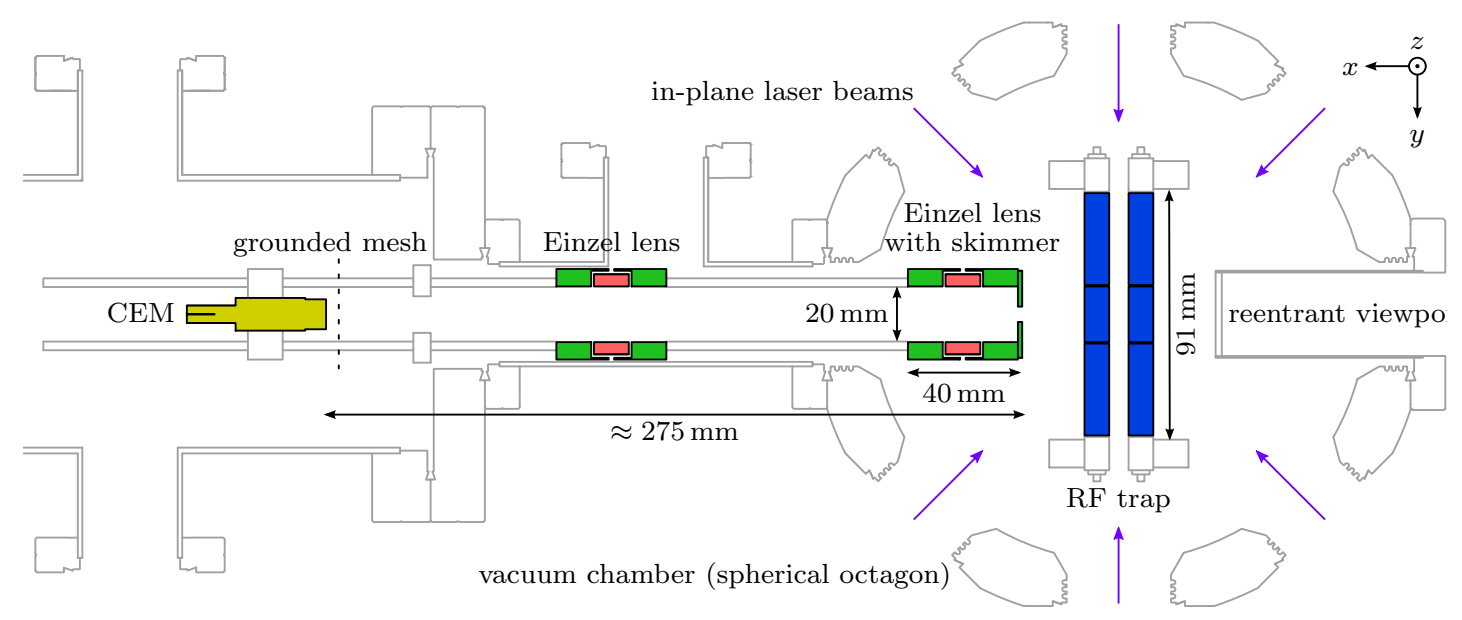

Figure 1. Schematic of the vacuum apparatus (top view). The linear segmented RF trap has cylindrical electrodes with a field radius of $R_{0}=6.85 \mathrm{~mm}$ and an electrode diameter of $9 \mathrm{~mm}$. The TOF drift tube is aligned perpendicularly to the axis of the RF trap to enable the radial extraction. Two Einzel lenses (red/green), one at the entrance of the drift tube and one roughly in the middle of the drift tube, focus the ions onto a channel electron multiplier (CEM, yellow), which is shielded by a stainless steel mesh (transmission $\sim 3 / 4$ ). The first Einzel lens has an additional skimmer with a $\approx 6$ mm diameter aperture (also green). Laser access for cooling of ions in the RF trap and an optionally overlapped magneto-optical trap for calcium atoms is possible from six in-plane directions and additional out-of-plane directions (not shown). The mounting structures and vacuum chamber (partially shown; gray lines) as well as the outer electrodes of the Einzel lenses (green) are grounded. The assembly is held under vacuum at a pressure of $\approx 10^{-9}$ mbar.

enter the drift tube in order to prevent a reduction of mass resolution.

Considering the cold preparation of the ion samples prior to their exctraction here, neither the defocussing during extraction nor the resulting ion loss at the skimmer seem to impose limitations. This is supported by the molecular-dynamics simulations presented in Ref. 1 which were performed without fields from the Einzel lenses and the experimental observation of reduced but still significant ion signals when turning off the Einzel lenses despite an acceptance angle of only $\sim 0.02$ (corresponding to a numerical aperture of $\sim 0.01$ ) of the CEM.

Einzel lenses, CEM and mesh are mounted on three stainless steel rods which allow for better pumping of the vacuum chamber as compared to a solid tube. The complete drift tube assembly fits into a standard 2.75 in $\mathrm{CF}$ nipple. In order to minimize stray fields close to the RF trap, the grounded outer Einzel lens electrodes encapsulate the inner electrode carrying high voltages. Additional constraints in the current setup require slight deviations from Ref. 14, in particular, the distance of the first Einzel lens to the RF trap is increased such that MOT laser beams with a diameter $\sim 1 \mathrm{~cm}$ are not clipped.

\section{Drive Unit}

The block diagram of the drive unit is given in Fig. 2 and a photograph is shown in Fig. 3. The entire unit is implemented on a single printed-circuit board (PCB). The incoming RF signal passes an RC-bandpass filter and is amplified by a preamplifier (Analog Devices
ADA4897-1) and power amplifier (MOSFET: Microsemi ARF460AG). The power amplifier is operated in class A mode to minimize distortion and drives the primary side of a toroidal transformer (ferrite: material $61, \mu=125$, 1.4" outer diameter). An impedance of only a few Ohms is chosen to limit the supply voltage of the amplifier to $24 \mathrm{~V}$ and the number of turns of the transformer to a few ten.

The secondary side of the transformer together with a trim capacitor, $\mathrm{PCB}$ traces, cables, the vacuum feedthrough, and the $\mathrm{RF}$ trap segment form a $L C$ resonator $(L \sim(0.5-1.0) \mathrm{mH}$ depending on the desired $\Omega$ and $C \sim 100 \mathrm{pF})$. The trim capacitor $((12-48) \mathrm{pF})$ allows for fine-tuning the resonance frequency and matching the frequencies of the respective drive units. While one end of the secondary winding of the transformer is connected to the segment of the RF trap, the other end is $\mathrm{RF}$ grounded via a capacitor $(\sim 20 \times C)$ which allows biasing of the RF trap segment with both a positive $U_{\mathrm{DC}}$ and $U_{\mathrm{HV}}$.

$U_{\mathrm{DC}}$ is applied via a low-pass filter and an HV diode (as a protection from $U_{\mathrm{HV}}$ ) to RF ground. Similarly, a $U_{\mathrm{HV}}$ pulse is applied to RF ground by activating a MOSFET (IXYS IXTH1N250) which is supplied by a HV power supply and bypassed with a capacitor $(1 \mu \mathrm{F})$. As a result of this design, not only the RF trap segment but the entire secondary side of the drive unit is biased with $U_{\mathrm{DC}}$ permanently and $U_{\mathrm{HV}}$ during pulsing. While the $\mathrm{RF}$ transformer naturally isolates the amplifier, any digital signals must be passed by digital isolators (Analog Devices ADUM series) to the secondary side and are supplied by a DC-DC converter. As the DC-DC converter 


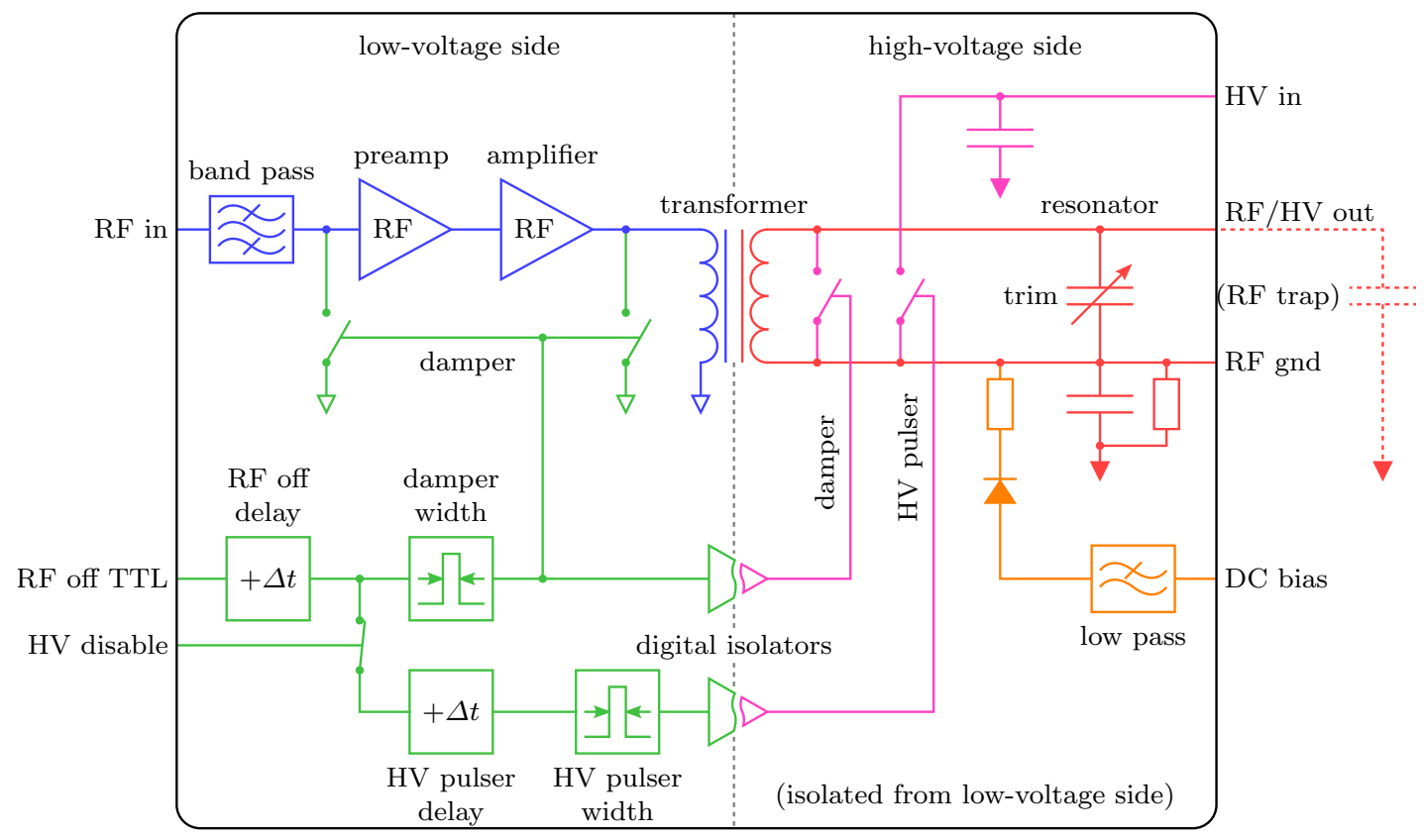

Figure 2. Block diagram of a drive unit. The PCB is divided into a low-voltage (left) and high-voltage (right) side, which are galvanically isolated from each other. The low-voltage side consists of RF amplifiers and primary winding of the RF transformer (blue) and timing and damping circuitry (green). The high-voltage side comprises the secondary winding of the transformer with capacitors forming the resonator (red), damping and HV pulsing circuitry (purple), and a $U_{\mathrm{DC}}$ bias supply (orange).

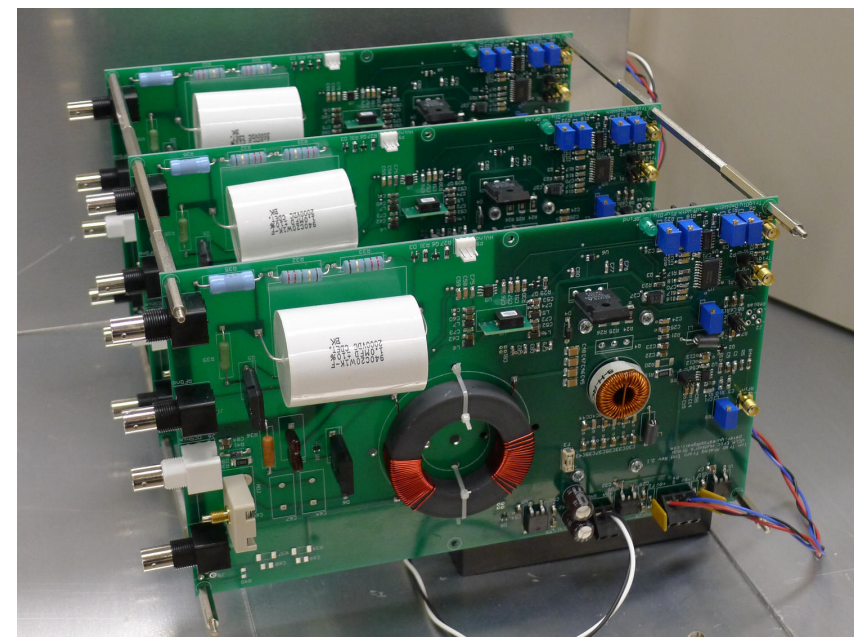

Figure 3. Photograph of three drive units assembled to fit into one 19" enclosure. The high-voltage side can be identified on left-hand side by the light green PCB material (because it has no ground plane) compared to the dark green low-voltage side. The PCBs stand on the heatsinks (partially visible behind the front $\mathrm{PCB}$ ) of their RF amplifier MOSFETs. Four copies of this assembly are required to operate the RF trap and TOFMS.

introduces noise on the RF drive around its switching frequency plus harmonics, a battery can optionally buffer the voltage and allows to switch off the DC-DC converter during experiments.

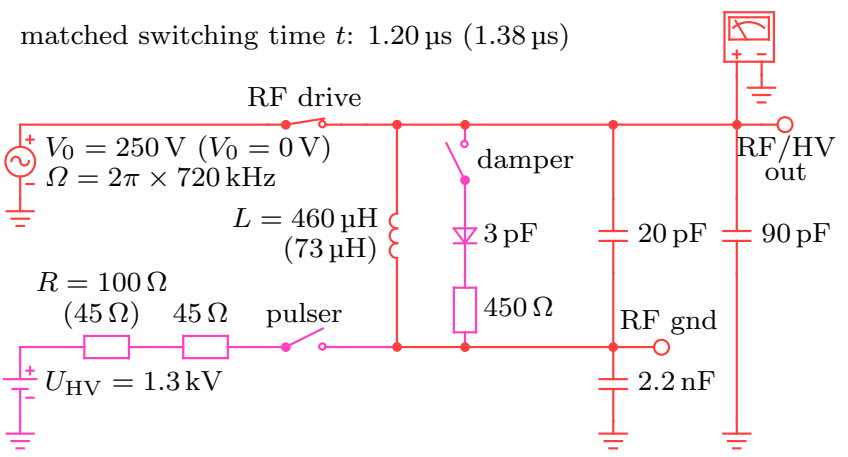

Figure 4. Simplified circuit diagram of the relevant components of a drive unit for simulation of the HV pulsing with Qucs [55] for an RF drive frequency $\Omega=\Omega_{<}=2 \pi \times 720 \mathrm{kHz}$. The RF drive starts with a positive zero-crossing at $t=0$ in the simulation. Switches change at time $t$ from open (closed) to closed (open) positions to disable RF drive and activate damping and HV pulsing, respectively. Values approximate $\Omega_{<}$drive units; deviating values for $\Omega_{>}$drive units are given in parenthesis. Prior to matching, both drive units use $R=100 \Omega$ and $t \approx 1.389 \mu \mathrm{s}$. Coarse matching leads to $R=45 \Omega$ for the $\Omega_{>}$drive units and the switching times given in the diagram.

Turning off the input RF signal to the resonant circuit would result in a ring-down of the RF voltage over a few $10 \mu \mathrm{s}$. Analogously, the application of the HV pulse would cause significant ringing on top of the HV pulses for extraction into the TOFMS. To remedy these effects, 


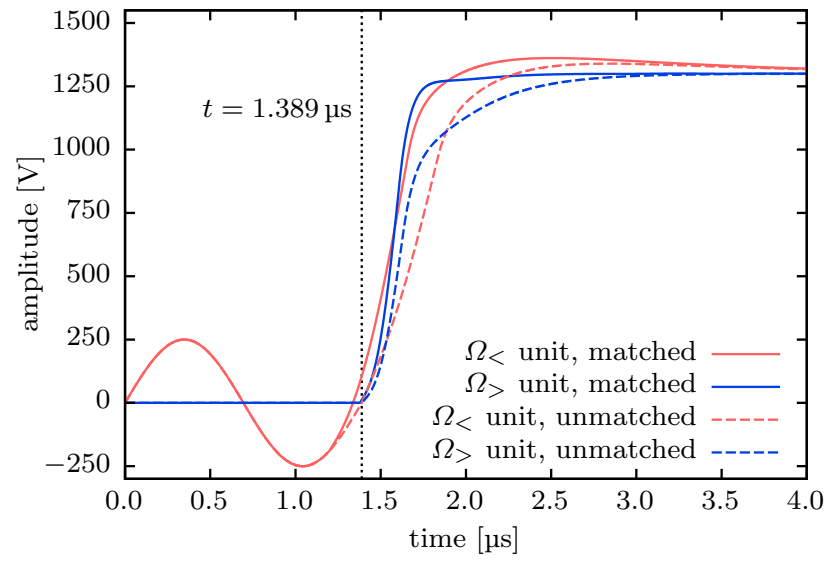

Figure 5. Simulated output voltages of the $\Omega_{<}$and $\Omega_{>}$drive units for the coarsely matched case (solid curves) compared to the values prior to matching (dashed curves); compare Fig. 4.

we implemented an active damping of the resonator on both its primary and secondary side using MOSFETs (IXYS IXTH02N250), which effectively shorts the windings of the RF transformer. A single input TTL signal initiates both the damping and the HV pulse. Logic circuitry allows adjustment of delay and duration of the damping and the HV pulse individually and is used to match the timings across all drive units.

Still, activating the damping and HV pulsing sequence at random times and, hence, random phases of the RF drive would result in ringing and varying slopes of the $\mathrm{HV}$ pulses. Hence, the control unit (see Sec. IID) contains circuitry to generate TTL signals which are synchronized with the RF drive phase to switch roughly at the zerocrossing of the RF drive.

Further, drive units belonging to $\Omega_{<}$and $\Omega_{>}$exhibit initially different $\mathrm{HV}$ pulsing characteristics caused by the different inductances, $L$, of their RF transformers. In order to assist matching, we perform a simulation with the circuit simulator Qucs [55]. As an example, the circuit diagram for the case $\Omega=\Omega_{<}=2 \pi \times 720 \mathrm{kHz}$ is presented in Fig. 4. Without matching, (1) both drive units use a resistor $R=100 \Omega$ to limit the HV pulsing current and (2) the HV pulses of both the $\Omega_{<}$and $\Omega_{>}$are activated at the positive zero-crossing of the RF drive (at time $t \approx 1.389 \mu \mathrm{s}$ in the simulation). The corresponding HV pulse forms are shown in Fig. 5 (dashed curves) and are in good agreement with the experimentally observed pulse forms.

Coarse matching can be achieved by two modifications: (1) Using $R=45 \Omega$ for the $\Omega_{>}$drive units increases the HV pulsing current and removes the "kink" in the pulse form at $\sim 3 / 4 U_{\mathrm{HV}}$, including the shallower slope. (2) Activating the pulses of the $\Omega_{<}$drive units sightly before the positive zero-crossing of the RF drive at $t=1.20 \mu \mathrm{s}$ leads to a steeper slope and approximates the HV pulses of the $\Omega_{>}$drive units better. (To match the timing, the HV pulses of the latter drive units are also activated slighly earlier at $t=1.38 \mu \mathrm{s}$.) The coarsely matched HV pulse forms are also shown in Fig. 5 (solid curves). The experimental system performance is discussed later (see Sec. III A and Fig. 9).

Matching using modification (2) removes the possibility of having a delay between turning off the RF drive and activating the pulses and, hence, "time-lag energy focussing" as described for the original Wiley-McLaren TOFMS [49] is not possible. However, this is permissible without sacrificing TOFMS resultion, because the ions will be sufficiently cold (usually laser cooled) in the intended experiments.

Drive unit PCBs (for the three segments of an electrode; see Fig. 3) are housed by threes in 19 " rack mount enclosures, which shield the sensitive RF amplifier circuits. Further, the design of the enclosures involves thermal management to keep the components, particularly those of the resonator, close to ambient temperature and minimize thermal drifts. As a result, the drive units have a sufficient passive stability such that no repeated matching is required.

\section{Control Unit}

The control unit has three main purposes: (1) it provides synchronized RF signals for the drive units; (2) it allows for computer control of the RF parameters (frequency, phase, amplitude) and certain experimental sequences (extraction into the TOF, loading of the RF trap via laser ablation); and (3) synchronization of the RF damping and HV pulses with the RF drive phase.

A block diagram of the control unit is depicted in Fig. 6 and a photograph in Fig. 7. The unit uses a microcontroller (Atmel ATmega 2560), which is connected over a serial-to-USB interface (FTDI FT232R) to a computer. The microcontroller is powered over USB and its digital inputs/outputs are galvanically isolated by digital isolators (Analog Devices ADUM series), which provides both reduction of noise and protection of the computer in case of a malfunction.

Four external digital inputs and four outputs allow interfacing with external TTL-compatible devices. All four inputs are assigned interrupts such that externally triggered events meet hard real-time criteria. As an example, one of the TTL inputs can be used to turn off the DDS channels.

The RF signals are generated by four direct digital synthesis (DDS) devices (Analog Devices AD9959) each having four channels. The DDS devices allow for a sample rate of $500 \mathrm{MSPS}$ and individual frequency, phase, and amplitude control of each channel. For simplicity, we utilize evaluation boards (Analog Devices AD9959/PCB).

Synchronization of the DDS devices is subject to three conditions. First, all four devices must receive the same reference clock (REFCLK) signal. This is achieved with a $500 \mathrm{MHz}$ reference oscillator (Crystek RFPRO33500.000), followed by an amplifier (Mini-Circuits ZX60- 


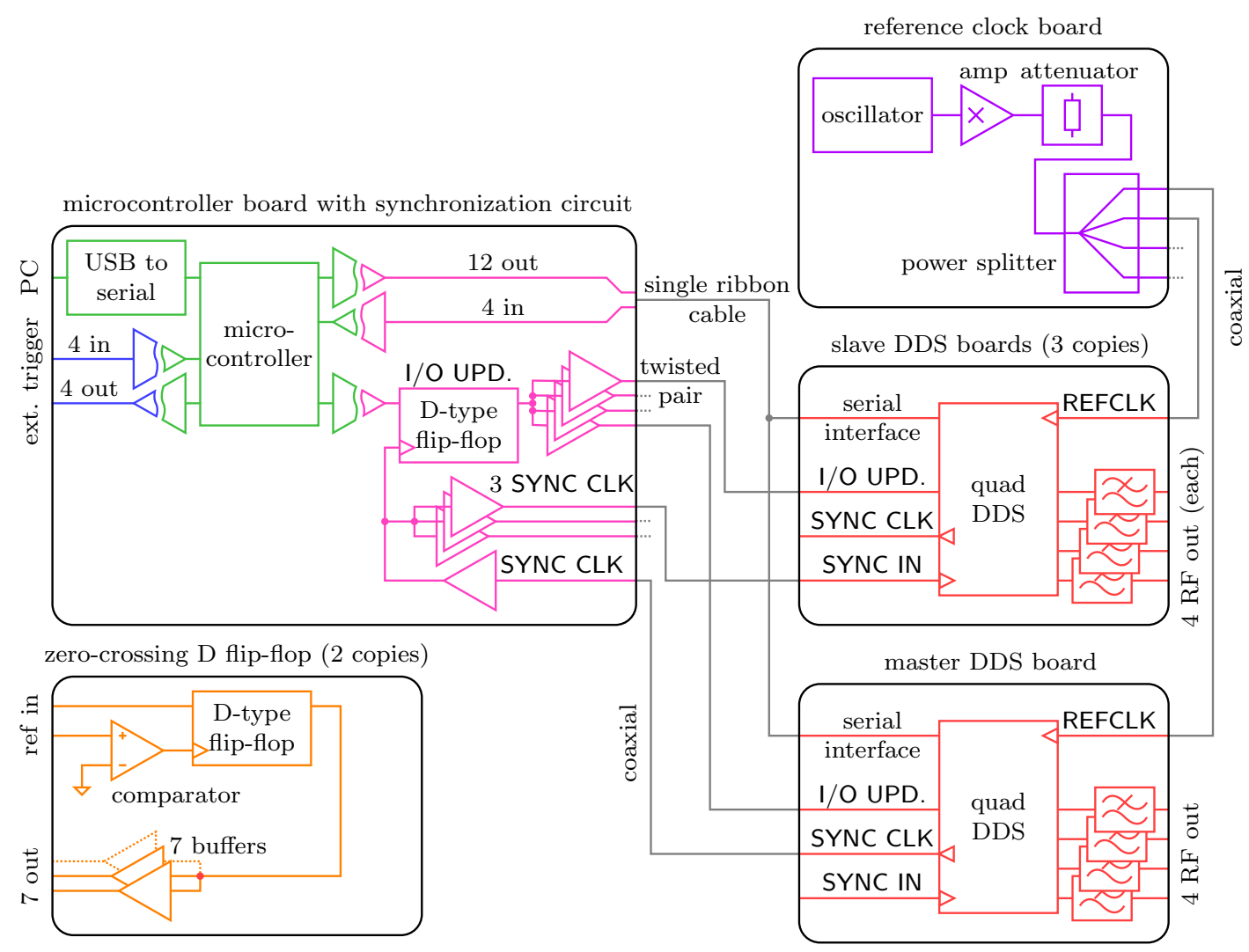

Figure 6. Block diagram of the control unit. The unit contains a microcontroller which is connected to a PC via USB (green) and possesses external trigger inputs and outputs (blue). The microcontroller can program four DDS devices (red) over an SPIcompatible serial interface. The DDS devices are synchronized by using the same reference clock (violet) and a synchronization circuit on the microcontroller PCB (purple). Two PCBs based on D-type flip-flops synchronize the TTL signal for initiating the HV pulses with the RF drive phase (orange). The microcontroller part is galvanically isolated from the rest of the control unit.

33LN), attenuators (Mini-Circuits VAT series), a power splitter (Mini-Circuits ZB4PD1-500), and coaxial cables of equal length.

Second, the synchronization clocks (SYNC CLK $=$ REFCLK/4 in our case) of the digital interface of the DDS devices must be synchronized across all four DDS devices. Without synchronization, the SYNC CLKs of the four DDS devices will have mutual phase differences of a random integer-multiple of $90^{\circ}$ upon initialization. We chose automatic mode synchronization and supply the buffered (Texas Instruments 74LVC541) SYNC CLK output of the master device to the SYNC IN of the slaves. This results in a deterministic phase difference of an integer-multiple of $90^{\circ}$ between master and slave devices upon initialization, which can be removed by programming certain registers of the slave DDS devices.

Third, the I/O UPDATE signal upon which data in the serial I/O buffer of the DDS devices are transferred into active registers must be synchronized with the SYNC CLK. The I/O UPDATE originates from the microcontroller and is initially unsynchronized, as the microcontroller has its own $16 \mathrm{MHz}$ clock. An edge-triggered Dtype flip-flop (Texas Instruments SN74LVC1G79) passes the unsynchronized signal upon the rising-edge of the master's SYNC CLK to the DDS devices and hence ensures an appropriately synchronized I/O UPDATE signal. (This is the reason for use of the master's SYNC CLK instead of SYNC OUT for synchronization, because the latter requires activation over the serial interface upon reset. This, however, would not be possible, because I/O UPDATE would not work without the clock for the flipflop.)

The digital interface between the microcontroller and DDS devices is serial peripheral interface (SPI) compatible. Each DDS device uses an individual chip select $(\overline{\mathrm{CS}})$ and serial data out (SDO) pin of the microcontroller; any other signal is connected in parallel across all DDS devices. This layout manages without active components (e.g. multiplexers) and limits the required number of wires. Further, the DDS devices feature a profile selection over four designated pins, which allows to switch between certain parameters within one clock cycle without reprogramming registers over the SPI interface. The corresponding pins are also wired in parallel across all DDS devices and, currently, enable us to turn off all DDS channels simultaneously. 


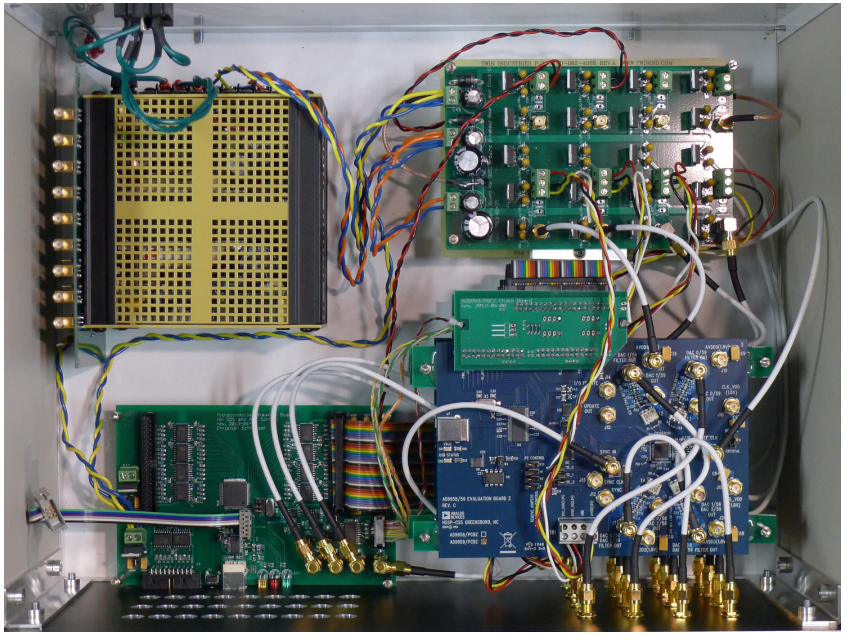

Figure 7. Photograph of the control unit. The microcontroller board with synchronization circuit (bottom left) is connected to a stack of four DDS boards (blue, bottom right). The serial interface is connected via the colored ribbon cable and small PCBs (upper left corner of DDS boards) to facilitate wiring and selection of $\overline{C S}$ and SDO. A supply (upper left) supplies a voltage regulator $\mathrm{PCB}$ (upper right) providing power for various components. The reference clock board is located below the voltage regulator board (upper right). The zero-crossing D-type flip-flop boards are normally located on top of the microcontroller board, but have been temporarily removed. (One is standing upright, left of the power supply.)

The last component of the control unit is a zerocrossing D-type flip-flop PCB for synchronizing the damping and HV pulse with the RF drive phase. Similar to the I/O UPDATE case, an edge-triggered D-type flip-flop (Texas Instruments SN74LVC1G79) passes an externally provided, unsynchronized trigger signal to the drive units upon the zero-crossing of a reference RF signal from a spare DDS channel using a comparator (Analog Devices ADCMP601). This translates the excellent phase control of the DDS channels to a control over the timing of the damping and HV pulsing. In total, we use two such zero-crossing D-type flip-flop PCBs with their own DDS reference channels to control the timining of the $\Omega_{<}$and $\Omega_{>}$drive units individually.

\section{E. Wiring and Measuring}

The outputs of the drive units are connected to the vacuum chamber over $\approx 175 \mathrm{~cm}$ long low-capacitance coaxial cables (RG-62, $42 \mathrm{pF} / \mathrm{m}$ ), which represent the largest single contribution to the total capacitance of the resonator. These cables allow for sufficient spatial separation between vacuum chamber and optical setup such that thermal issues are prevented.

The cables have $75 \Omega$ mini-SMB connectors and are plugged into one of four assemblies on the vacuum chamber (see Fig. 8). Each assembly consists of a PCB
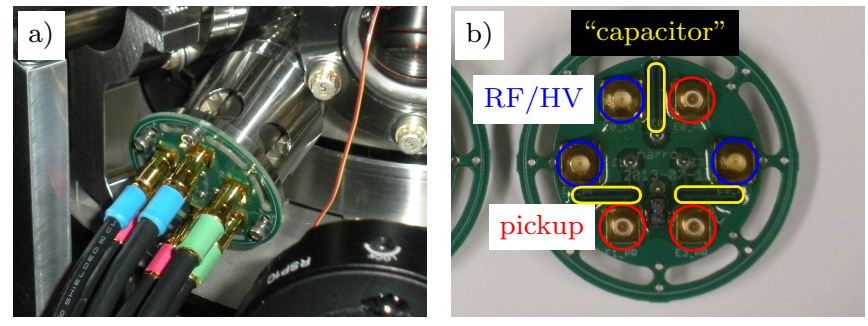

Figure 8. (a) Feedthrough assembly for one electrode of the $\mathrm{RF}$ trap. The PCB is attached with a mechanical mount on top of a four-wire feedthrough and provides the interface between wires and SMB connectors. (b) Top view of the PCB. Three SMB connectors are used to supply the RF/HV voltages, while the other three SMB connectors are used to measure the input voltages via capacitors implemented by close-by PCB traces.

with mechanical mount, which is attached to a standard 1.33 "-CF four-wire vacuum feedthrough. The PCBs interface the wires of the feedthroughs with the help of receptacles (Mill-Max 0492-0-15-15-13-14-04-0) to SMB connectors and provide the ground connection to the vacuum chamber. On the vacuum side, three wires per feedthrough are connected to the three segments of an electrode (leaving one wire per feedthrough unused).

The interface PCBs also include capacitive pickup traces close to $\mathrm{RF} / \mathrm{HV}$ traces, which sample a small fraction of the $\mathrm{RF} / \mathrm{HV}$ voltage supplied to the segments. The pickup signal allows the measurement of the input voltages at each segment with a probe ratio of $\approx 1000: 1$ (as measured with an oscilloscope with $1 \mathrm{M} \Omega$ impedance and typical cable lengths). The ratios are calibrated against an $\mathrm{HV}$ probe (Agilent 10076B) to better than $1 \%$ (relative). In order to prevent arcing, in particular across the pickup traces, the PCBs are sealed with conventional two-component epoxy adhesive and tested with proof voltages exceeding $3 \mathrm{kV}$.

\section{DISCUSSION}

\section{A. System Performance}

Typical output voltages as measured on the inner four segments of the RF trap are given in Fig. 9. The RF amplitudes can be matched using such pickup signals to at least the $1 \%$ level and their mutual phase offsets can be minimized below $\approx 0.1 \%$. The HV slopes can be matched to time differences $<5 \mathrm{~ns}$ and show jitters $<$ 5 ns. Ultimately, RF phase offsets or amplitudes are finetuned using feedback on trapped ion Coulomb crystals; individual $U_{\mathrm{HV}}$ values are optimized for highest TOFMS detection yields and mass resolution.

The detailled analysis of the performance of the TOFMS is described elsewhere [1]. An example TOF mass spectrum after ablating the $\mathrm{Yb}$ target and laser cooling one of the $\mathrm{Yb}$ isotopes is depicted in Fig. 10 (see 


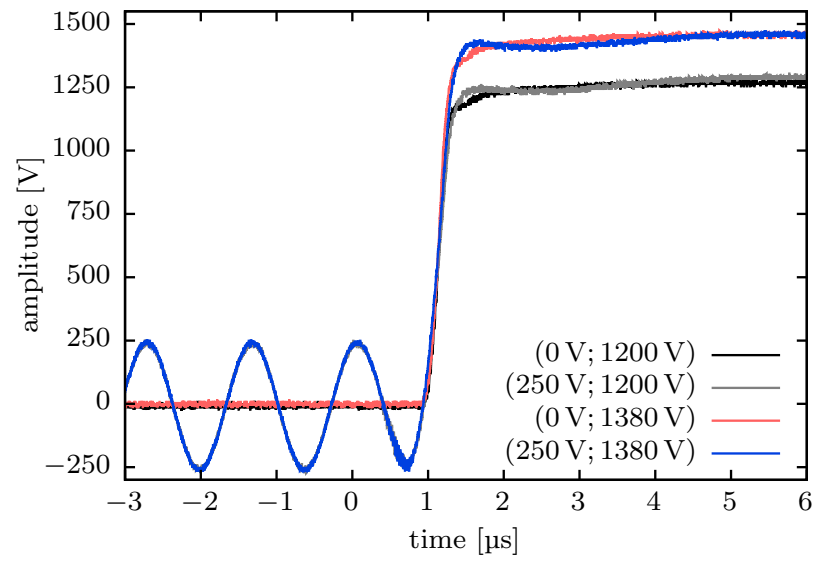

Figure 9. Typical RF voltages and $\mathrm{HV}$ pulses $\left(V_{\mathrm{RF}} ; U_{\mathrm{HV}}\right)$ at $\Omega=2 \pi \times 720 \mathrm{kHz}$ as measured at the inner four segments of the RF trap (see Sec. II E) and recorded with an oscilloscope. The time $t=0$ refers to the the trigger of the drive units and the offset of the HV pulses depends on settings of the timing circuitry.

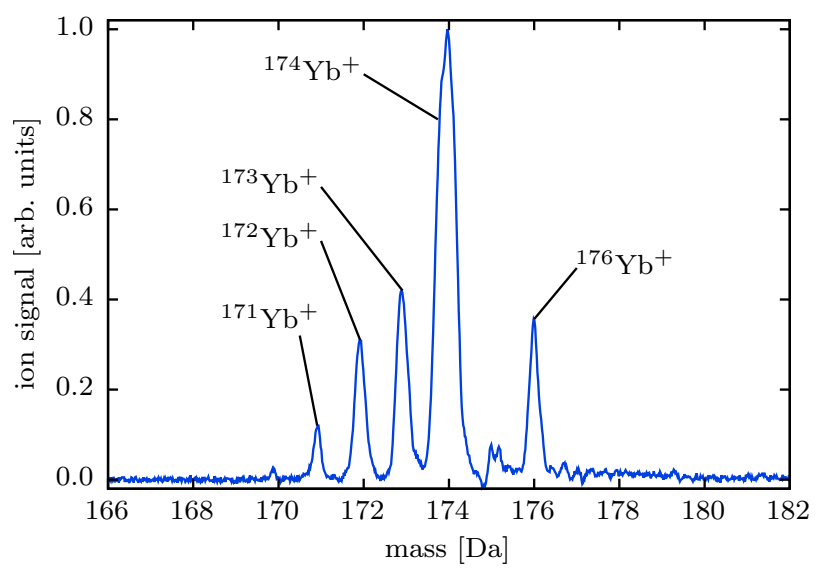

Figure 10. TOFMS spectrum for trapped $\mathrm{Yb}$ ablation products (see Ref. 1 for more details). Samples consisting of $\approx 1000$ ions are loaded into the RF trap and ${ }^{174} \mathrm{Yb}^{+}$is laser cooled. The curve represents the average of 20 spectra.

Ref. 1 for details). The spectrum shows resolved peaks for all natural isotopes of $\mathrm{Yb}$. The peak heights do not represent the natural abundances of the isotopes, because the sample preparation is biased towards the laser-cooled isotope. Additionally, the laser-cooled ions can more efficiently sympathetically cool heavier ions compared to lighter ones. The small peak around $175 \mathrm{Da}$ is likely an electronic artifact and/or due to $\mathrm{YbH}^{+}$, as there is no naturally occuring ${ }^{175} \mathrm{Yb}$. Still, provided the ion detector is not saturated, the peak heights represent the abundance of the different isotopes in the sample.

The TOFMS can be calibrated absolutely with various ion species which are at our disposal $\left(\mathrm{Ba}^{+}, \mathrm{BaCl}^{+}\right.$, $\left.\mathrm{Yb}^{+}\right)$. The low jitter of the timing circuits and the re- producibility of the extraction voltages including their phase relation to the $\mathrm{RF}$ drive voltages leads to a variation of the flight time of the same mass within the limits imposed by the mass resolution. This allows to average TOF traces measured over several days without sacrificing isotopic mass resolution.

\section{B. Future Improvements}

The design choice of a segmented linear RF trap with twelve individual drive units complicates the matching of $\mathrm{RF}$ drive voltages and HV pulses. Further, the freedom in choice of RF phase and amplitude for each segment is restricted, because the mutual capacitance between the segments leads to strong coupling of the three resonators. Future versions will use a non-segmented linear RF trap with continuous RF electrodes and external DC endcap electrodes, which reduces the number of drive units to four.

Further, the CEM ion detector has a comparatively small aperture and the ion signal is generated in a funnellike structure along the propagation direction of the ions, which can impact the mass resolution. Replacing it with a micro-channel plate (MCP) promises an improved detection limit catching ions with trajectories further away from the drift tube axis, potentially higher mass resolution because of its flatness, and, especially, improved linearity.

Currently, the slope of the HV pulses is intentially limited to a $10 \%-90 \%$ rise time of $\approx 250 \mathrm{~ns}$ by the resistor $R$. This reduces the stress on the switching components and promises a longer lifetime, however, it might also limit the mass resolution. A (slight) improvement of mass resolution might be achieved by using larger $\mathrm{HV}$ pulse currents without the resistor.

Ions in the RF trap have typical secular frequencies of $\omega=2 \pi \times(5-100) \mathrm{kHz}$. The ions' secular motion in the RF trap can be heated by various mechanism [56], for example, by low-frequency electronic noise around $\omega$. This noise is reduced by the abovementioned filtering and battery buffering. Additionally, noise on the RF drive in a region $\Omega \pm \omega$ can heat the ions' secular motion, which is particularly important to consider when using digital RF sources such as a DDS device. The frequency spectrum of the drive units shows an average noise floor at the $\leq-90 \mathrm{dBc}$ level at $\approx \Omega \pm \omega$, however, due to the digital character of the DDS devices, peaks with amplitudes of typically $\leq-70 \mathrm{dBc}$ are present. The location and amplitude of these peaks depends strongly on the generated output RF frequency. While current experiments have not been limited by heating of the RF trap, the noise might become a concern in future experiments. As a large number of indepently controllable RF sources is required for the setup described in this manuscript, it appears unrealistic to use multiple high-quality RF generators typically used with RF traps. As a cheaper solution, analog oscillators could be phase-locked to the outputs of 
the DDS devices and provide low-noise RF sources without losing the phase and frequency control provided by the DDS devices.

In principle, the demonstrated RF trap with integrated TOFMS is suited for work on heavier molecules. Singly-charged molecules of masses $m \sim 1000$ Da could be trapped, sympathetically cooled [45], and resolved by the TOFMS, which opens up experiments on a variety of volatile organic compounds, the amino acids, explosive agents, some peptides, and heavier biomolecules such as nucleic acids. The mass range could be significantly extended by permitting multiply-charged molecules as in Ref. 57. For such experiments, alternative soft ionization techniques, such as electrospray ionization (ESI) $[58,59]$ and matrix-assisted laser desorption/ionization (MALDI) [60-62], are readily available and would require only small modifications of the presented vacuum chamber.

Lastly, the described experimental setup allows the overlap a Ca magneto-optical trap (MOT) with $\mathrm{Ba}^{+}$, $\mathrm{BaCl}^{+}$, and $\mathrm{Yb}^{+}$ions in the $\mathrm{RF}$ trap. The Ca MOT leads to a production of undesired $\mathrm{Ca}^{+}$and $\mathrm{Ca}_{2}^{+}$ions [34], which have an only slightly lower mass than $\mathrm{Ba}^{+}$. The operation of the RF trap in a regime that is unstable for $\mathrm{Ca}^{+} / \mathrm{Ca}_{2}^{+}$requires a high Mathieu- $q$ parameter [56] for all (desired and undesired) ions. Thus, it dictates the comparatively low drive frequency $\Omega_{<}$and comparatively high $\mathrm{RF}$ amplitude $V_{0}$. A high Mathieu- $q$ parameter in turn leads to increased heating rates and problems for large ion Coulomb crystals. To tackle this problem, we will explore operation of the RF trap with different $\mathrm{RF}$ drive frequencies being used simultaneously to engineer unstable regions in the stability diagram for certain masses. The electronics described in this manuscript already allow such a dual-frequency mode with the two drive frequencies $\Omega_{<}$and $\Omega_{>}$and, with it, we have demonstrated production of ion Coulomb crystals. Additionally, such a dual-frequency mode could also be advantageous for more exotic trapping scenarios of ions of largely different masses [63]. For other applications, operation at a lower $q$ parameter should lead to improved trapping conditions and performance.

\section{ACKNOWLEDGEMENT}

We thank Alexander Dunning and Prateek Puri for critically reading the manuscript. This work was supported by the ARO Grant No. W911NF-15-1-0121, ARO MURI Grant No. W911NF-14-1-0378, and NSF Grant No. PHY-1205311.
[1] C. Schneider, S. J. Schowalter, K. Chen, S. T. Sullivan, and E. R. Hudson, Phys. Rev. Appl. 2, 034013 (2014).

[2] X. Tong, A. H. Winney, and S. Willitsch, Phys. Rev. Lett. 105, 143001 (2010).

[3] E. R. Hudson, Phys. Rev. A 79, 032716 (2009).

[4] T. Schneider, B. Roth, H. Duncker, I. Ernsting, and S. Schiller, Nat. Phys. 6, 275 (2010).

[5] P. F. Staanum, K. Højbjerre, P. S. Skyt, A. K. Hansen, and M. Drewsen, Nat. Phys. 6, 271 (2010).

[6] A. K. Hansen, O. O. Versolato, L. Kłosowski, S. B. Kristensen, A. Gingell, M. Schwarz, A. Windberger, J. Ullrich, J. R. C. López-Urrutia, and M. Drewsen, Nature 508, 76 (2014).

[7] C.-Y. Lien, C. M. Seck, Y.-W. Lin, J. H. Nguyen, D. A. Tabor, and B. C. Odom, Nat. Commun. 5, 4783 (2014).

[8] W. G. Rellergert, S. T. Sullivan, S. J. Schowalter, S. Kotochigova, K. Chen, and E. R. Hudson, Nature 495, 490 (2013).

[9] Y.-P. Chang, K. Długołęcki, J. Küpper, D. Rösch, D. Wild, and S. Willitsch, Science 342, 98 (2013).

[10] M. V. DePalatis and M. S. Chapman, Phys. Rev. A 88, 023403 (2013).

[11] S. Willitsch, M. T. Bell, A. D. Gingell, S. R. Procter, and T. P. Softley, Phys. Rev. Lett. 100, 043203 (2008).

[12] N. Deb, L. L. Pollum, A. D. Smith, M. Keller, C. J. Rennick, B. R. Heazlewood, and T. P. Softley, Phys. Rev. A 91, 033408 (2015).

[13] K. Chen, S. J. Schowalter, S. Kotochigova, A. Petrov, W. G. Rellergert, S. T. Sullivan, and E. R. Hudson, Phys. Rev. A 83, 030501 (2011).
[14] S. J. Schowalter, K. Chen, W. G. Rellergert, S. T. Sullivan, and E. R. Hudson, Rev. Sci. Instrum. 83, 043103 (2012).

[15] C. M. Seck, E. G. Hohenstein, C.-Y. Lien, P. R. Stollenwerk, and B. C. Odom, Spectroscopic Tests of Fundamental Physics, J. Mol. Spectrosc. 300, 108 (2014).

[16] P. Puri, S. J. Schowalter, S. Kotochigova, A. Petrov, and E. R. Hudson, J. Chem. Phys 141, 014309 (2014).

[17] A. Dunning, A. Petrov, S. J. Schowalter, P. Puri, S. Kotochigova, and E. R. Hudson, J. Chem. Phys. 143, 124309 (2015).

[18] K.-K. Ni, H. Loh, M. Grau, K. C. Cossel, J. Ye, and E. A. Cornell, Spectroscopic Tests of Fundamental Physics, J. Mol. Spectrosc. 300, 12 (2014).

[19] D. J. Larson, J. C. Bergquist, J. J. Bollinger, W. M. Itano, and D. J. Wineland, Phys. Rev. Lett. 57, 70 (1986).

[20] T. Baba and I. Waki, Jpn. J. Appl. Phys. 35, L1134 (1996).

[21] R. Rugango, J. E. Goeders, T. H. Dixon, J. M. Gray, N. B. Khanyile, G. Shu, R. J. Clark, and K. R. Brown, New J. Phys. 17, 035009 (2015).

[22] W. W. Smith, O. P. Makarov, and J. Lin, J. Mod. Opt. 52, 2253 (2005).

[23] A. T. Grier, M. Cetina, F. Oručević, and V. Vuletić, Phys. Rev. Lett. 102, 223201 (2009).

[24] A. Härter and J. Hecker Denschlag, Contemp. Phys. 55, 33 (2014).

[25] C. Sias and M. Köhl, in Quantum Gas Experiments: Exploring Many-Body States, Cold Atoms, Vol. 3, edited by P. Törmä and K. Sengstock (Imperial College Press, 
Singapore, 2014) Chap. 12, pp. 267-291.

[26] S. Willitsch, arXiv 1401.1699v1, 1 (2014), 1401.1699.

[27] C. Zipkes, S. Palzer, C. Sias, and M. Köhl, Nature 464, 388 (2010).

[28] S. Schmid, A. Härter, and J. Hecker Denschlag, Phys. Rev. Lett. 105, 133202 (2010).

[29] C. Schneider, M. Enderlein, T. Huber, and T. Schaetz, Nat. Photonics 4, 772 (2010).

[30] C. Schneider, M. Enderlein, T. Huber, S. Dürr, and T. Schaetz, Phys. Rev. A 85, 013422 (2012).

[31] M. Enderlein, T. Huber, C. Schneider, and T. Schaetz, Phys. Rev. Lett. 109, 233004 (2012).

[32] T. Huber, A. Lambrecht, J. Schmidt, L. Karpa, and T. Schaetz, Nat. Commun. 5, 5587 (2014).

[33] C. Zipkes, S. Palzer, L. Ratschbacher, C. Sias, and M. Köhl, Phys. Rev. Lett. 105, 133201 (2010).

[34] S. T. Sullivan, W. G. Rellergert, S. Kotochigova, K. Chen, S. J. Schowalter, and E. R. Hudson, Phys. Chem. Chem. Phys. 13, 18859 (2011).

[35] W. G. Rellergert, S. T. Sullivan, S. Kotochigova, A. Petrov, K. Chen, S. J. Schowalter, and E. R. Hudson, Phys. Rev. Lett. 107, 243201 (2011).

[36] F. H. J. Hall, M. Aymar, N. Bouloufa-Maafa, O. Dulieu, and S. Willitsch, Phys. Rev. Lett. 107, 243202 (2011).

[37] L. Ratschbacher, C. Zipkes, C. Sias, and M. Köhl, Nat. Phys. 8, 649 (2012).

[38] S. T. Sullivan, W. G. Rellergert, S. Kotochigova, and E. R. Hudson, Phys. Rev. Lett. 109, 223002 (2012).

[39] F. H. J. Hall and S. Willitsch, Phys. Rev. Lett. 109, $233202(2012)$

[40] I. Sivarajah, D. S. Goodman, J. E. Wells, F. A. Narducci, and W. W. Smith, Phys. Rev. A 86, 063419 (2012).

[41] A. Makarov, E. Denisov, A. Kholomeev, W. Balschun, O. Lange, K. Strupat, and S. Horning, Anal. Chem. 78, 2113 (2006).

[42] W. Paul and H. Steinwedel, Z. Naturforsch. Teil A 8, 448 (1953).

[43] M. Drewsen, A. Mortensen, R. Martinussen, P. Staanum, and J. L. Sørensen, Phys. Rev. Lett. 93, 243201 (2004).

[44] A. Ostendorf, C. B. Zhang, M. A. Wilson, D. Offenberg, B. Roth, and S. Schiller, Phys. Rev. Lett. 97, 243005 (2006).

[45] S. Schiller and C. Lämmerzahl, Phys. Rev. A 68, 053406 (2003).
[46] C. B. Zhang, D. Offenberg, B. Roth, M. A. Wilson, and S. Schiller, Phys. Rev. A 76, 012719 (2007).

[47] S. Kahra, G. Leschhorn, M. Kowalewski, A. Schiffrin, E. Bothschafter, W. Fuß, R. de Vivie-Riedle, R. Ernstorfer, F. Krausz, R. Kienberger, and T. Schaetz, Nat. Phys. 8, 238 (2012).

[48] R. Alheit, T. Gudjons, S. Kleineidam, and G. Werth, Rapid Comm. Mass Spectrom. 10, 583 (1996).

[49] W. C. Wiley and I. H. McLaren, Rev. Sci. Instrum. 26, 1150 (1955).

[50] C. L. Jolliffe, "Mass spectrometer with radial ejection," (1996), patent, US 5576540

[51] J. Franzen, "Method and device for orthogonal ion injection into a time-of-flight mass spectrometer," (1998), patent, US 5763878.

[52] N. Kjærgaard and M. Drewsen, Phys. Plasmas 8, 1371 (2001).

[53] N. Kjærgaard, K. Mølhave, and M. Drewsen, Phys. Rev. E 66, 015401 (2002).

[54] A. A. Makarov, E. V. Denisov, and A. Kholomeev, "Rf power supply for a mass spectrometer," (2009), patent, US 7498571.

[55] The Qucs team, "Qucs: Quite universal circuit simulator," Open Source Software (2013), version 0.0.17.

[56] D. J. Wineland, C. Monroe, W. M. Itano, D. Leibfried, B. E. King, and D. M. Meekhof, J. Res. Natl. Inst. Stand. Technol. 103, 259 (1998).

[57] D. Offenberg, C. B. Zhang, C. Wellers, B. Roth, and S. Schiller, Phys. Rev. A 78, 061401 (2008).

[58] M. Yamashita and J. B. Fenn, J. Phys. Chem. 88, 4451 (1984).

[59] J. B. Fenn, M. Mann, C. K. Meng, S. F. Wong, and C. M. Whitehouse, Science 246, 64 (1989).

[60] M. Karas, D. Bachmann, and F. Hillenkamp, Anal. Chem. 57, 2935 (1985).

[61] K. Tanaka, H. Waki, Y. Ido, S. Akita, Y. Yoshida, T. Yoshida, and T. Matsuo, Rapid Commun. Mass Spectrom. 2, 151 (1988).

[62] F. Hillenkamp, M. Karas, R. C. Beavis, and B. T. Chait, Anal. Chem. 63, 1193A (1991).

[63] D. Trypogeorgos and C. J. Foot, arXiv 1310.6294v2, 1 (2013), 1310.6294. 


\section{graphical_abstract}
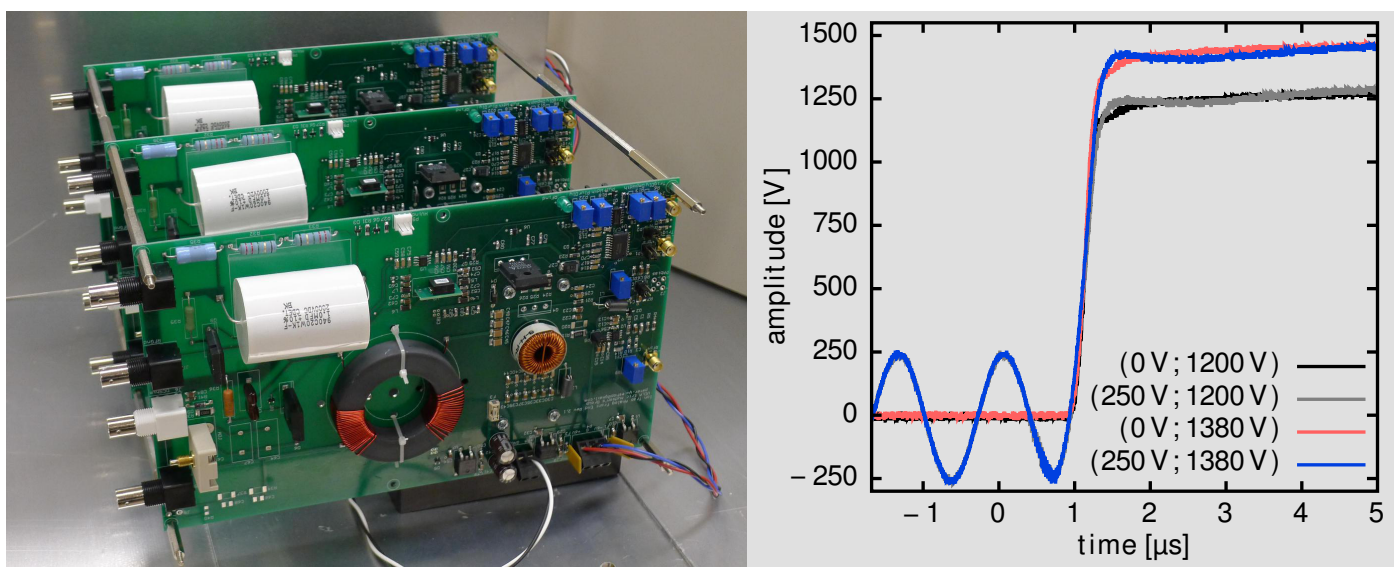